\title{
Deutschsprachige Präventionsprogramme für Essstörungen - Ein systematisches Review
}

\section{German Prevention Programs for Eating Disorders - A Systematic Review}

Autoren

Mara Pickhardt, Luise Adametz, Felicitas Richter, Bernhard Strauß, Uwe Berger

Institut

Institut für Psychosoziale Medizin und Psychotherapie, Universitätsklinikum Jena

\section{Schlüsselwörter}

Essstörung, Präventionsprogramm, systematisches Review, Risikofaktoren

Key words

eating disorder, prevention program, systematic review, risk factors

eingereicht $\quad 24.02 .2017$

akzeptiert $\quad 14.11 .2017$

Bibliografie

DOI https://doi.org/10.1055/s-0043-124766

Online-Publikation: 13.2.2018

Psychother Psych Med 2019; 69: 10-19

(c) Georg Thieme Verlag KG Stuttgart · New York

ISSN 0937-2032

Correspondence

Luise Adametz, M.Sc.

Institut für Psychosoziale Medizin und Psychotherapie

Universitätsklinikum Jena

Stoystraße 3

07740 Jena

luise.adametz@med.uni-jena.de

$\Theta$ Die Tab. 2-4 finden Sie unter https://doi.org/10.1055/ s-0043-124766.

\section{ZUSAMMENFASSUNG}

Im deutschsprachigen Raum existiert mittlerweile eine Vielzahl primärer und sekundärer Präventionsprogramme für Essstörungen, jeweils entwickelt für unterschiedliche Zielgruppen und Kontexte. Ziel dieser Arbeit war eine systematische Zusammenfassung der Inhalte und Ergebnisse primärer und sekundärer deutschsprachiger Präventionsprogramme sowie die Integration der Studienergebnisse. Dies soll medizinischem Fachpersonal aber auch Psychologen, Lehrkräften und Sozialarbeitern einen umfassenden Überblick bei der Auswahl wirksamer und gut evaluierter Programme bieten. Die systematische Literaturrecherche ergab 22 primär- und sekundärpräventive Präventionsprogramme für Essstörungen, davon waren 11 wissenschaftlich evaluiert. Die Programme sind entweder für Schulen, das Internet oder ambulante Gruppen konzipiert. Die Mehrzahl der Studien weist auf eine Reduktion von Gewichts- und Figursorgen und Schlankheitsstreben sowie auf eine Erhöhung des (Körper-) Selbstwerts in der Gesamtstichprobe oder in der Risikogruppe hin. Eine Reduktion des auffälligen Essverhaltens hingegen scheint nur bei Risikogruppen oder sekundärpräventiven Programmen möglich. Alle Programme verändern mind. eine Zielvariable bedeutsam. Sekundäre Präventionsprogramme erzielten höhere Effektstärken als primärpräventive. Limitationen der Studien und Implikationen für zukünftige Forschung werden diskutiert.

\section{ABSTRACT}

In the past years a considerable amount of primary and secondary prevention programs for eating disorders was developed in German speaking countries. However, up to now there has been no systematic review of contents and evaluation studies. The main objective of the present systematic review is to identify and outline German prevention programs for eating disorders. This should facilitate the selection of appropriate and effective interventions for medical experts, other professionals and teachers. A systematic literature research was conducted and 22 German-language primary and secondary prevention programs were identified. Half of them were evaluated. The programs were conducted either in school, on the internet or in a group setting. The findings show that throughout almost all programs a reduction in weight and shape concerns and drive for thinness as well as an increase of (body) self-esteem could be observed in either the total sample or the high-risk sample. However, programs were inconsistently effective in reducing disordered eating behavior in the target population. All studies were effective in reducing at least one risk factor. Overall, higher effect sizes were found for secondary prevention programs than for primary prevention programs. Lastly, limitations of the studies and suggestions for future prevention efforts are discussed. 


\section{Einleitung}

Essstörungen zählen zu seltenen, jedoch schweren psychischen Erkrankungen. Die Lebenszeitprävalenzen aller Essstörungen liegen bei 5,9\% (weibliche Erwachsene) bzw. bei 4,8\% (weibliche Jugendliche): Anorexia nervosa (AN) 0,9 bzw. 1,7\%; Bulimia nervosa (BN) 3,5 bzw. 0,8\%; Binge-Eating-Störung (BED) 3 bzw. 2,3\% [1,2]. Deutlich häufiger treten subklinische Essstörungen, auffälliges Essverhalten und einzelne Essstörungssymptome in der Adoleszenz auf. In dem deutschlandweiten repräsentativen Kinder- und Jugendgesundheitssurvey (KiGGS) berichteten 21,9\% der 11-17-Jährigen auffälliges Essverhalten [3]. Aus klinischen Interviews hervorgehend, ist bei 13,7\% der 14-24-Jährigen vom Auftreten einzelner Kernsymptome einer Essstörung auszugehen, wobei Mädchen deutlich häufiger betroffen sind als Jungen [4]. Diese Zahlen sind bedeutsam, da auffälliges Essverhalten als bedeutender Risikofaktor für die Entwicklung einer Essstörung gilt. Bereits im subklinischen Bereich stehen Essstörungen im Zusammenhang mit Verschlechterungen des psychosozialen Funktionsniveaus, internalisierenden (z. B. Angst und Depression) und externalisierenden (z. B. delinquentes Verhalten) Verhaltensauffälligkeiten sowie dem Risiko persistierender Symptomatik [4, 5]. Präventive Maßnahmen sind daher notwendig, um Risikofaktoren zu reduzieren und Schutzfaktoren aufzubauen [4]. Einflussreiche Risikofaktoren sind Schlankheitsstreben, Gewichts- und Figursorgen, Perfektionismus, weibliches Geschlecht, negativer Selbstwert, negatives Körperbild und Diäthalten [6, 7]. Schutzfaktoren sind ein hohes Selbstwertgefühl, ein positives Körperbild, hohe Selbstwirksamkeitserwartung und Kompetenzen wie Konfliktfähigkeit und Medienkompetenz [8, 9].

Präventive Maßnahmen untergliedern sich in primäre und sekundäre Maßnahmen [12]. Primäre Prävention soll dem Wachstum von Risikofaktoren vorbeugen, um Inzidenzraten zu senken. Die Zielgruppe des primär universellen Ansatzes ist dabei eine große Bevölkerungsgruppe mit und ohne vorhandene Risikofaktoren. Die Zielgruppe des primär selektiven Ansatzes sind Personen ohne individuelle Risikofaktoren, aber mit biologischen, soziokulturellen oder psychologischen Risikofaktoren. Ein Beispiel für diesen Ansatz ist ein Programm speziell für Mädchen, da diese aufgrund ihres Geschlechts bereits ein erhöhtes Erkrankungsrisiko aufweisen. Sekundär indizierte Präventionsansätze sollen das Erkrankungsrisiko von Personen mit individuell erhöhtem Risiko reduzieren. In dieser Zielgruppe treten bereits einzelne Symptome auf, ohne dass ein Vollbild einer klinisch relevanten Störung vorliegt.

In den vergangenen 20 Jahren wurden bereits viele Präventionsstrategien entwickelt und erfolgreich evaluiert [10-14]. Universelle und selektive Präventionsansätze zeigten sich mit kleinen bis mittleren Effektstärken wirksam in der Reduktion bekannter Risikofaktoren für Essstörungen [14]. Merkmale erfolgreicher Programme waren u. a. ein selektiver Ansatz für Hochrisikogruppen, ein interaktiver Aufbau, ein Programm, dass mehrere Lektionen umfasste, geschlechterspezifische Lektionen, die Durchführung von ausgebildeten Experten und die Behandlung von Programminhalten wie Körperakzeptanz und Induktion von Dissonanz. 51 \% der Programme zeigten sich wirksam darin, mind. einen Risikofaktor für Essstörungen zu reduzieren [12]. Weitere wesentliche Merkmale erfolgreicher Programme sind die Durchführung im Gruppensetting, die theoretische Fundierung und die Bearbeitung relevanter Themen wie Ernährung, Medienkompetenz, Körperakzeptanz und Selbstbewusstsein [10,13-15]. Die Hinweise auf das passende Alter der Zielgruppe sind heterogen. Während Stice und Kollegen auf eine gesteigerte Effektivität von Präventionsprogrammen bei Jugendlichen ab 15 Jahren hinwiesen, fanden Yager und Kollegen erfolgreichere Ergebnisse bei Jugendlichen zwischen 12 und 13 Jahren $[12,13]$.

Die oben geschilderten Erkenntnisse beruhen auf Meta-Analysen englischsprachiger Präventionsprogramme für Essstörungen. Eine Übertragung der Programme auf das deutsche Schulsystem erscheint aufgrund sozialer, politischer und kultureller Unterschiede nicht empfehlenswert [16]. Im deutschsprachigen Raum wurden daher bereits einige Präventionsprogramme entwickelt [17]. Eine systematische Übersichtsarbeit und Integration der Studienergebnisse zu deutschsprachigen Präventionsprogrammen liegt aktuell nicht vor. Die Vielzahl der verfügbaren Programme erschwert jedoch die Auswahl. Eine Systematisierung hilft Berufsgruppen wie Psychologen, Lehrkräften oder Sozialarbeitern, die primäre und sekundäre Präventionsprogramme in unterschiedlichen Kontexten anbieten (z. B. Schulen, Beratungsstellen), ein geeignetes, wirksames und gut evaluiertes Präventionsprogramm zu wählen. Ziel dieser Arbeit ist eine systematische Zusammenfassung der Inhalte und Evaluationsergebnisse primärer und sekundärer Präventionsprogramme für Essstörungen im deutschsprachigen Raum. Dies bietet Interessierten einen umfassenden Überblick und unterstützt insbesondere die adressatengerechte Auswahl wirksamer Programme.

\section{Methode}

Das vorliegende systematische Review orientiert sich an den Preferred Reporting Items for Systematic Reviews and Meta-Analyses (Prisma-Richtlinien) [18]. Ziele, Einschlusskriterien und Methoden wurden vorab in einem Studienprotokoll spezifiziert und registriert (PROSPERO Reg. No.: CRD42016042094) [19].

\section{Auswahlkriterien}

Die Einschlusskriterien der Studien entsprechen den PICOS- Richtlinien. Betrachtet werden P: Participants (Teilnehmer); I: Intervention; C: Comparison (Vergleich); O: Outcome (Ergebnisse) und S: Study Design (Studiendesign) [18] ( Tab. 1). Zusätzliche Einschlusskriterien waren: deutsche oder englische Sprache, publizierte und unpublizierte Literatur und deutsche Programmsprache.

\section{Informationsquellen und Literatursuche}

Es wurde eine systematische Literaturanalyse in den elektronischen Datenbanken PsycINFO, PubMed, und Web of Science durchgeführt, welche sich in 3 relevante, miteinander kombinierte Bereiche gliederte: Diagnose (z. B. eating disorder * , disordered eating, eating behaviour, subthreshold eating, binge eating), Intervention (z. B. primary prevention, secondary prevention, selective prevention, preventive intervention) und Design (z. B. control-trial, experimental design, effectiveness). Die letzte Suche erfolgte am 07.07.2016. Außerdem wurden Forschende und Arbeitsgruppen relevanter Arbeiten mit der Anfrage nach unpublizierten Studien kontaktiert sowie die Referenzlisten bereits identifizierter Publikationen geprüft. 
- Tab. 1 Einschlusskriterien der Studien (entsprechend PICOS-Richtlinien).

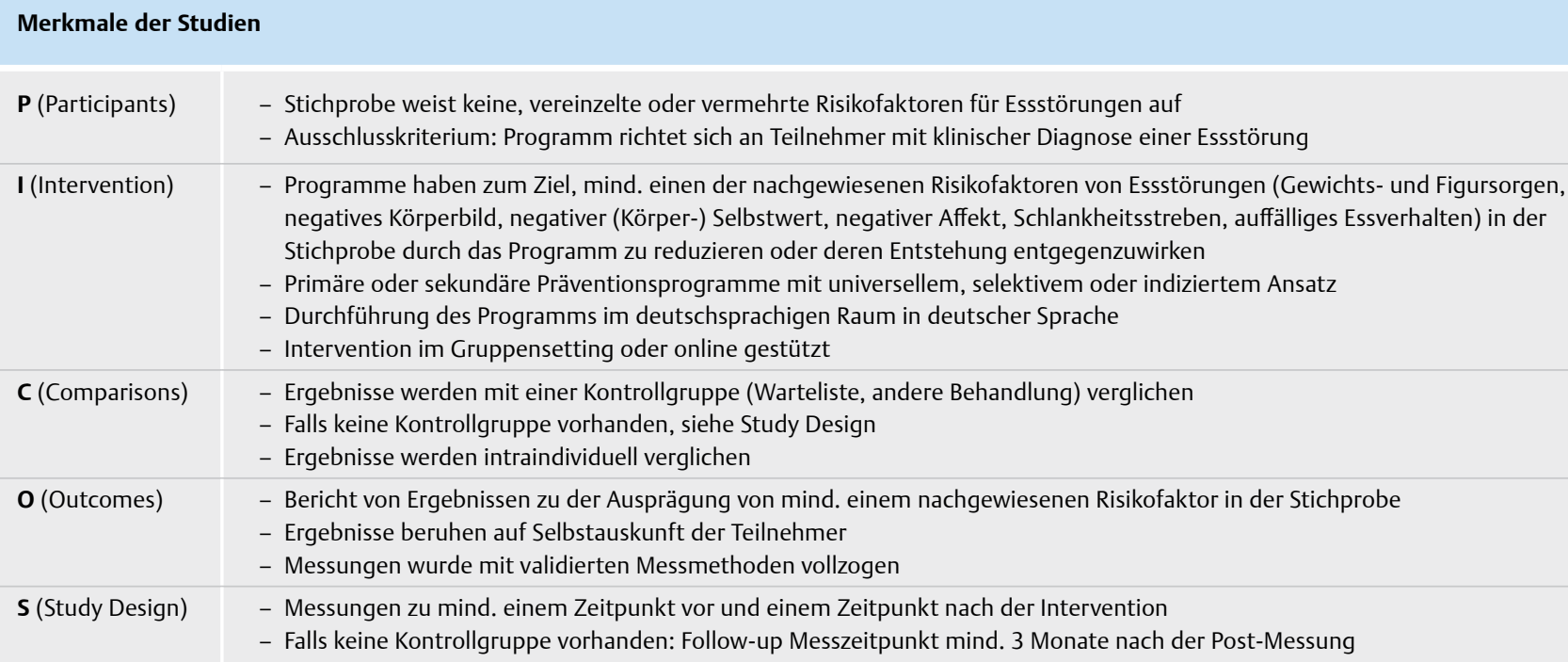

\section{Studienselektion und Prozess der Datengewinnung}

Eine erste Auswahl relevanter Studien erfolgte per Screening der Studienzusammenfassungen. Der weitere Auswahlprozess fand anhand der Volltexte statt. Mithilfe des erstellten Kodierplans, wurden die Informationen der eingeschlossenen Studien extrahiert. Zur Integration verschiedener Ansätze in den Analyse- und Kodierungsprozess waren im Kodierungsprozess mehrere Forscher beteiligt. Aufgetretene Unterschiede wurden per Diskussion und Konsensbildung behoben. Folgende Aspekte wurden erfasst: Merkmale der Studie (Titel, Autoren, Jahr, Land), Stichprobe (Stichprobengröße, Alter und Geschlecht), Intervention (Interventionsansatz, Häufigkeit und Länge der Intervention, theoretischer Hintergrund, Setting der Intervention), Methodik (Studiendesign, Follow-up) Ergebnisse (gemessene Zielvariablen). Publikationen, die sich auf denselben Datensatz beziehen, wurden nur einmal berücksichtigt.

\section{Verzerrungsrisiken der einzelnen Studien}

Das Verzerrungsrisiko der einzelnen Studien wurde auf Basis der PRISMA-Richtlinien hinsichtlich der relevantesten Aspekte zusammengefasst. Die Kategorien zur qualitativen Einschätzung des Verzerrungsrisikos lauten:

- Vollständige Ergebnisdarstellung

- Follow-up Messung mind. 3 Monate nach der Post Messung

- Manualisiertes und theoretisch fundiertes Programm

- Messungen mit validierten Messmethoden (Kriterium erfüllt, wenn jeder der Tests validiert wurde)

- Drop-out-Rate kleiner als 15\% (von Prä-/Basline-Messung zu Post-Messung)

- Berechnung der statistischen Power

\section{Synthese der Ergebnisse}

Eine narrative systematische Übersicht der bestehenden deutschsprachigen Programme gibt Auskunft über wesentliche Charakteristika sowie Merkmale erfolgreicher und weniger erfolgreicher Pro- grammbestandteile. Im quantitativen Teil erfolgt die Berechnung der standardisierten Mittelwertdifferenz Hedges g als Effektstärke, welche einen Vergleich der Zielvariablen jeder Studie zwischen Interventions- und Kontrollgruppe und über Studien hinweg ermöglicht [20]. Beim Fehlen einer Kontrollgruppe erfolgte die Effektstärkenberechnung innerhalb einer Gruppe (intraindividueller Vergleich). Wenn Daten vorlagen, wurden zusätzlich Follow-up Effektstärken berechnet. Bei Verwendung mehrerer Messinstrumente zur Erhebung einer Zielvariablen wurde eine zusammenfassende Effektstärke gebildet. Die Berechnung erfolgte mit dem Programm Comprehensive Meta Analysis (CMA, Version 3.0). Positive Effektstärken deuten auf einen Vorteil der Interventions- gegenüber der Kontrollgruppe hin, negative Effektstärken deuten auf einen Nachteil der Interventions- gegenüber der Kontrollgruppe hin. Die Größe des Effekts der Effektstärke g wird anlehnend an Cohens d interpretiert mit einem kleinen Effekt ab 0,20, einem mittleren Effekt ab 0,50 und einem großen Effekt ab 0,80 [21].

\section{Ergebnisse}

\section{Übersicht der Programme}

Die Literaturrecherche ergab insgesamt 22 deutschsprachige Programme zur Primär- oder Sekundärprävention von Essstörungen ( $\triangleright$ Tab. 2, im Internet). Davon kamen 20 Programme aus Deutschland, ein Programm aus Österreich [22] und ein Programm aus der Schweiz [23]. Als primärpräventive Programme wurden identifiziert: Aufklärung und Prävention [24, 25], Body Talk [26], Bauchgefühl [27], Essenslust und Körperfrust [28], every Body [29], Healthy Teens @ School [22], Jugend mit Biss [30], MaiStep (Mainzer Schultraining zur Essstörungsprävention [31]), POPS (Potsdamer Prävention an Schulen [32]), PriMa (Primärprävention von Magersucht) [33-36], Student Bodies [37-40], Torera (Primärprävention Bulimie und Binge Eating) $[41,42]$, das Trainingsprogramm an 
Schulen [43] und Young Es[s]prit [44]. Zu den sekundärpräventiven Programmen zählen: ESS-KIMO (Klärendes Internetprogramm zur Steigerung der Veränderungsmotivation bei Essstörungen [45]), ES[S]PRIT [46], ein unbenanntes Präventionsprogramm von Buddeberg-Fischer und Kollegen [23], ein unbenanntes Präventionsprogramm von Köster und Kollegen [47, 48], ProYouth (Promotion of young people's mental health through technology enhanced personalization of care [49-51] und die beiden Programme Student Bodies + [52] und Student Bodies AN [53].

\section{Studienauswahl und -merkmale}

Die Literatursuche in den Datenbanken und weiteren Quellen ergab 1640 Treffer, von denen 18 Studien eingeschlossen wurden. Der Studienauswahlprozess ist in $\mathbf{A b b} \mathbf{1}$ dargestellt. Diese 18 Studien evaluierten 11 Programme. Es existieren weitere 11 Programme. Diese wurden jedoch nicht in die Ergebnissynthese eingeschlossen, da entweder keine Evaluationsstudien vorlagen $(n=8)$ oder die Evaluationsstudien nicht den Einschlusskriterien entsprachen ( $n=3), z$. B. wurden nicht validierte Messinstrumente benutzt, ausschließlich Lehrer befragt oder nur Daten zu Inanspruchnahme oder Kosteneffektivität berichtet [27, 30, 49, 54].

Die Eigenschaften der 18 eingeschlossenen Studien inklusive Studiendesign und Stichprobencharakteristika sind $>$ Tab. 3 (im Internet) zu entnehmen. Insgesamt wurde die Wirkung von Präventionsprogrammen für Essstörungen bisher an einer Gesamtstichprobengröße von N = 5852 Personen untersucht (primärpräventive Programme $n=5170$, sekundärpräventive Programme $n=682$ ). 6 Studien wiesen eine Stichprobengröße von $N<100$ auf. 7 Studien wiesen ein randomisiert kontrolliertes Design auf, eine
Studie hatte keine Kontrollgruppe [53]. 10 der eingeschlossenen Studien waren Pilotstudien. 5 der eingeschlossenen Studien sind unpublizierte Arbeiten. 7 der Studien hatten keinen Follow-upMesszeitpunkt. Der Anteil weiblicher Personen in der Gesamtstichprobe lag mit 74,11\% höher als der Anteil männlicher Personen. Das mittlere Alter der Probanden lag zwischen 12 und 27 Jahren. Dabei wiesen Studien zu primärpräventiven Programmen in der Regel deutlich jüngere Personen auf als Studien zu sekundärpräventiven Programmen. Bei diesen wurden mit Ausnahme vom Präventionsprogramm von Buddeberg-Fischer et al. ausschließlich erwachsene Probanden untersucht [23].

Die eingeschlossenen Studien erhoben folgende Zielvariablen (verwendete Messinstrumente sind in Klammern dargestellt): Inzidenz von Essstörungen ( $k=2)$, Auffälliges Essverhalten ( $k=16)$ (Eating-Attitudes-Test (EAT-26-D) [55]; SCOFF, [56]; Eating Disorder Examination Questionnaire (EDE-Q) [57]; Eating Disorders Inventory (EDI-2) [58]), Figurunzufriedenheit $(k=10)$ (Figurzeichnungen des Kids Eating Disorder Survey (KEDS) [59]; Body Dissatisfaction in EDI-2, [58]), Gewichts- und Figursorgen ( $k=8)$ (Weight and Shape Concerns Scale (WCS), [60]; Weight Concern in EDE-Q, [57]; Shape Concern in EDE-Q [57]), Depressivität $(k=2)$ (Beck Depression Inventar (BDI), [61]), Schlankheitsstreben $(k=10)$ (Drive for Thineness in EDI-2, [58]; Drive for Thinness in EDE-Q, [57]), (Körper-) Selbstwert $(k=10)$ (Fragebogen zur Beurteilung des eigenen Körpers (FBeK), [62]; Rosenberg Self-Esteem Scale (RSES), [63]; Self-Description-Questionaire (SDQ-III-G), [64]; State-Self-EsteemScale (SSED), [65]) und Wissen $(k=9)$ (Selbstentwickelte Skala zur Erfassung des Wissens zu AN, [35], Wissenstest, [66]; Fragebogen zu gesunder Ernährung und Essstörungen, [67]).

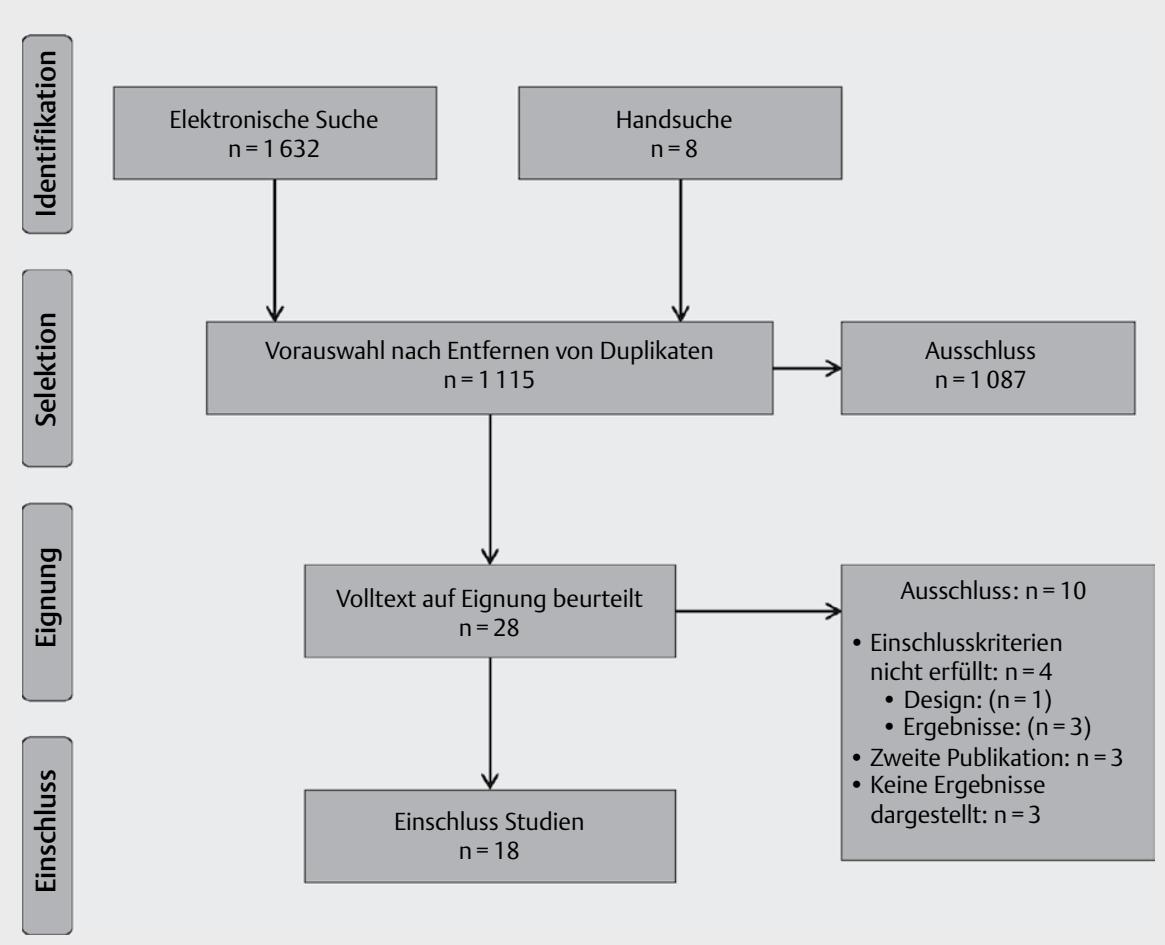

Abb. 1 Studienauswahlprozess 


\section{Risiko der Verzerrung innerhalb der Studien}

Insgesamt wiesen die Studien ein mittleres Verzerrungsrisiko auf ( $\vee$ Tab. 3 im Internet). Jede gelistete Studie wies mind. ein Verzerrungsrisiko auf. Dabei waren Drop-out-Raten über $15 \%(k=11)$ und die fehlende Berechnung und Angabe der statistischen Power $(k=13)$ besonders häufige Verzerrungsrisiken.

\section{Ergebnisse der einzelnen Studien und Synthese der Ergebnisse}

- Tab. 4 (im Internet) beinhaltet die berechneten Effektstärken (Hedges g) aller eingeschlossenen Studien. Gelegentlich traten nicht signifikante Ergebnisse auf, welche jedoch aufgrund ihrer Effektstärke von klinischer Bedeutung sind. Unabhängig von der statistischen Signifikanz werden daher alle Effekte ab einem Hedges $g \geq 0,2$ als bedeutsame Interventionseffekte interpretiert. Insgesamt war jedes Präventionsprogramm effektiv darin, mind. eine Zielvariable zu beeinflussen. Sekundärpräventive Programme waren in den meisten Fällen erfolgreicher auch mehr als eine Zielvariable positiv zu beeinflussen. Wurden Follow-up-Zeiträume untersucht, so waren die gefundenen positiven Effekte in der Regel stabil bis zum letzten Messzeitpunkt.

\section{Inzidenz von Essstörungen}

Zwei der eingeschlossenen Studien untersuchten die Inzidenzraten von Essstörungen nach Programmteilnahme. Für das Programm MaiStep zeigten sich in der 12-Monats-Katamnese signifikant weniger Fälle mit partieller Essstörungssymptomatik bei Präventionsteilnehmern im Vergleich zu Kontrollprobanden [31]. Für das Programm Young Es[s]prit konnte in der 12-Monats-Katamnese nicht eindeutig gezeigt werden, dass die Inzidenzrate in der Präventionsgruppe geringer war als in der psychoedukativen Kontrollgruppe [44].

\section{Auffälliges Essverhalten}

16 der 18 eingeschlossenen Studien untersuchten die Variable auffälliges Essverhalten. Programme mit primärpräventivem Ansatz zeigten meist keine signifikanten Effekte auf das Essverhalten der Teilnehmer und Teilnehmerinnen der Gesamtstichprobe (PriMa $[33,35]$, Torera [41, 42], Trainingsprogramm an Schulen [43], Student Bodies [38]). In Risikogruppen konnte jedoch eine Reduktion auffälligen Essverhaltens mit Effektstärken im kleinen bis mittleren Bereich gezeigt werden (PriMa [34], Torera [42], Kein Titel (Buddeberg-Fischer et al.) [23]). Die Studienergebnisse der sekundärpräventiven Präventionsprogramme zeigten eine Reduktion auffälligen Essverhaltens in den Gruppen mit Programmteilnahme (Student Bodies [39, 52, 53], ESS-KIMO [45], Kein Titel (Köster) [47]) mit Ausnahme von 2 Studien $[23,48]$.

\section{Figurunzufriedenheit}

Zehn der 18 Studien untersuchten die Wirkung von Präventionsprogrammen auf die Figurunzufriedenheit der Teilnehmerinnen und Teilnehmer. Studien zu Programmen mit primärpräventivem Ansatz zeigten keine Effekte auf die Figurunzufriedenheit in der Gesamtstichprobe (MaiStep [31], PriMa [33], Student Bodies [38, 40], Torera [41]). Jedoch zeigte sich bei Risikogruppen und in den sekundärpräventiven Programmen eine Reduktion der Figurunzufriedenheit im kleinen Effektstärkenbereich (PriMa [34], Torera [41], Kein Titel (Köster) [47, 48], Student Bodies [52]).
Gewichts- und Figursorgen

Acht der 18 Studien untersuchten die Variable Gewichts- und Figursorgen. Mit kleinen bis großen Effektstärken wirkte Student Bodies in allen Post- und Follow-up-Erhebungen positiv auf Gewichtsund Figursorgen [38-40, 53]. Das Gruppenprogramm von Köster $[47,48]$ zeigte sich ebenfalls mit kleinen bis mittleren Effekten wirksam, Gewichts- und Figursorgen in der Zielgruppe zu reduzieren. Nur eine Studie zeigte keine bedeutsame Veränderung der Gewichts- und Figursorgen in der Gruppe nach Programmteilnahme (ESS-KIMO [45]).

\section{Allgemeine Psychopathologie}

Zwei Studien zum Programm Student Bodies untersuchten die Variable Depressivität $[52,53]$. Das Programm erwies sich mit Effektstärken im kleinen bis mittleren Bereich als wirksam darin, Depressivität in der Gesamtstichprobe und in den Hochrisikogruppen zu reduzieren. Diese Effekte blieben überwiegend auch zum Followup Messzeitpunkt erhalten. Buddeberg-Fischer [23] evaluierten darüber hinaus die allgemeine Psychopathologie. Deren Programm erwies sich als nicht wirksam in der Reduktion der allgemeinen Psychopathologie in der Gesamtstichprobe sowie der Hochrisikogruppe.

\section{Schlankheitsstreben}

Zehn der 18 Studien erfassten die Auswirkungen der Teilnahme an den Präventionsprogrammen auf die Variable Schlankheitsstreben. Die Mehrheit der Programme war wirksam darin, das Schlankheitsstreben sowohl in der Risikogruppe als auch in Gesamtstichproben zu reduzieren, wobei die kleinen bis mittleren Effekte im Wesentlichen bis zur Follow-up Messung erhalten blieben [33, 34, 39, 40, $47,48,52,53]$. Lediglich 2 Studien konnten keinen bedeutsamen Einfluss ihrer Programme auf das Schlankheitsstreben der untersuchten Stichprobe zeigen $[31,38]$.

\section{(Körper-) Selbstwert}

Zehn der 18 Studien untersuchten den Einfluss der Präventionsprogramme auf die Variablen Selbstwert oder Körperselbstwert. Das Primärpräventionsprogramm PriMa erwies sich effektiv in der Verbesserung des Körperselbstwerts in der Risikogruppe und der Gesamtstichprobe mit kleinen Effektstärken [33-35]. Auch 8 Jahre nach der Intervention wiesen die Programmteilnehmerinnen einen signifikant stabileren Körperselbstwert als die Kontrollprobandinnen auf [36]. Ebenfalls signifikante Effekte mit kleinen Effektstärken zeigten sich in der Evaluation des Programms Torera. Mädchen, die sowohl an PriMa als auch Torera teilnahmen, profitierten besonders hinsichtlich der Verbesserung des Körperselbstwerts $[41,42]$. Das Trainingsprogramm an Schulen erwies sich mit überwiegend kleinen bis mittleren Effektstärken wirksam darin, den Selbstwert in der Stichprobe zu erhöhen [43]. Zwei sekundärpräventive Programme zeigten eine Verbesserung des Selbstwertes mit Effektstärken im kleinen Bereich (ESS-KIMO, [45], Kein Titel (Köster) $[47,48])$.

\section{Wissen}

Neun der 18 Studien untersuchten den Einfluss von Präventionsprogrammen auf das essstörungsspezifische Wissen. Hierbei zeigten sich alle primär- und sekundärpräventiven Programme erfolgreich darin, essstörungsspezifisches Wissen in den Zielgruppen mit 
Programmteilnahme aufzubauen. Die Effektstärken lagen dabei im kleinen, mittleren und großen Effektstärkenbereich und die Effekte konnten im Wesentlichen bis zum Follow-up Zeitpunkt erhalten bleiben (PriMa [34], Student Bodies [38, 39, 52], Torera [41], Trainingsprogramm an Schulen [43], Kein Titel (Köster) [47, 48]).

\section{Diskussion}

\section{Zusammenfassung}

Zur Prävention von Essstörungen im deutschsprachigen Raum existieren eine Reihe primär- und sekundärpräventiver Programme für unterschiedliche Zielgruppen und Kontexte. Dieses Review fasst systematisch die Inhalte und Studienergebnisse evaluierter Präventionsprogrammen für Essstörungen zusammen. Somit soll die Auswahl eines wirksamen und geeigneten Programms erleichtert werden.

Elf der 22 identifizierten Programme sind wissenschaftlich evaluiert worden. Alle Programme beinhalten interaktive Elemente (z. B. Gruppendiskussionen, Rollenspiele, Filmbeispiele), keines war ausschließlich psychoedukativ. Jedes Präventionsprogramm beeinflusste mind. eine Zielvariable effektiv. Sekundärpräventive Programme waren in den meisten Fällen erfolgreicher, auch mehr als eine Zielvariable positiv zu beeinflussen. Hierbei ist anzumerken, dass Primärprävention bei noch unbelasteten, jungen Zielgruppen stattfindet und darauf abzielt, dem Wachstum von Risikofaktoren über die Zeit vorzubeugen. Aufgrund der unterschiedlichen Ausgangswerte von primären und sekundären Zielgruppen erscheinen sekundärpräventive statistisch somit meist effektiver. Zur Erfassung der Wirksamkeit primärpräventiver Maßnahmen eigenen sich daher insbesondere Langzeiterhebungen. Wurden Follow-up-Zeiträume untersucht, so waren die gefundenen positiven Effekte häufig auch stabil bis zum letzten Messzeitpunkt - ein vielversprechender Hinweis auf nachhaltige Effektivität von Prävention. Die Effektstärken weisen, trotz der nicht immer statistisch signifikanten Effekte in den Stichproben, auf klinisch bedeutsame Veränderungen infolge der Teilnahme an Präventionsprogrammen hin.

Präventionsprogramme für Essstörungen haben das Ziel die Inzidenz von Essstörungen zu reduzieren, indem sie Risikofaktoren abbauen und Schutzfaktoren stärken. Nur 2 der 18 Studien untersuchten jedoch gezielt die Inzidenzrate von Essstörungen nach Programmteilnahme. Die diesbezüglichen Ergebnisse der beiden primär präventiven Programme waren heterogen, was keine gesicherte Aussage zulässt [31,44]. Auffälliges Essverhalten gilt als bedeutender Risikofaktor und ist die am häufigsten untersuchte Zielvariable der Studien. Eine Reduktion auffälligen Essverhaltens fand sich hier eher bei Risikogruppen und sekundärpräventiven Programmen. Diese Ergebnisse ordnen sich in die internationale Literatur ein, wobei $29 \%$ der Programme auffälliges Essverhalten reduzierten [12]. Auch auf Figurunzufriedenheit wirken die deutschsprachigen Programme v. a. bei Risikogruppen und Stichproben in sekundärpräventiven Programmen. Sieben von 8 Studien, die Auswirkungen auf Gewichts- und Figursorgen untersuchten, zeigten eine bedeutsame Reduktion in den Interventionsgruppen im Vergleich zu den Kontrollgruppen. Das Schlankheitsstreben war in 8 von 10 Studien in der Interventionsgruppe reduziert. Hinsichtlich des (Körper-) Selbstwertes wurde in 9 von 10 Studien eine bedeutsame Verbes- serung bei den Präventionsteilnehmern erzielt. Zwei Studien untersuchten den Einfluss auf Depressivität und stellten dabei eine Reduktion nach Programmteilnahme fest. Essstörungsspezifisches Wissen zeigte nach Programmteilnahme in allen Studien einen bedeutsamen Anstieg.

Eindeutige Hinweise auf Programmmerkmale, die den Erfolg der Präventionsprogramme beeinflussen, wurden nicht gefunden. Auffallend war eine inhaltliche und methodische Ähnlichkeit der Programme. Internetbasierte Programme und schulbasierte Programme waren vergleichbar effektiv. Das Alter der Stichprobe in den Studien variierte stark. Vielversprechende Ergebnisse zeigten sich aber sowohl bei jüngeren als auch älteren Zielgruppen. Internationale Meta-Analysen fanden ebenfalls Wirksamkeit bei jüngeren [13] als auch älteren Teilnehmern und Teilnehmerinnen [12]. Für gesicherte Wirksamkeitsaussagen sind längere Untersuchungszeiträume notwendig. V. a. bei jüngeren, noch unbelasteten Stichproben bestünde andernfalls die Gefahr, Präventionseffekte nicht zu detektieren.

Zusammengefasst sind die Effekte für Programme mit primärem Präventionsansatz in der Gesamtstichprobe kleiner als in der Risikogruppe oder bei Programmen mit sekundärem Präventionsansatz. Dies ist aufgrund des unterschiedlichen Ausmaßes vorhandener Risikofaktoren zum ersten Messzeitpunkt zu erwarten. Eine zusätzliche Verringerung der Ausgangswerte erscheint aufgrund des Bodeneffektes unwahrscheinlich [68]. Wahrscheinlicher ist die Aufrechterhaltung positiver Ausgangswerte. Die Langzeituntersuchung des primärpräventiven Programms PriMa demonstriert diesen Effekt. Acht Jahre nach der Intervention wies die Interventionsgruppe einen signifikant stabileren Körperselbstwert als die Kontrollgruppe auf [36]. Die Untersuchung von Risikogruppen zeigte Präventionseffekte in die gewünschte Richtung. Dieser Befund untermauert Erkenntnisse nationale und internationale Präventionsforschung $[12,13,15,69]$.

\section{Limitationen}

Eine wesentliche Limitation der eingeschlossenen Evaluationsstudien stellen die Einschränkungen in der methodischen Qualität der Studien dar ( $\vee$ Tab. 3 im Internet). Alle eingeschlossenen Studien wiesen mind. ein Verzerrungsrisiko auf, die Mehrheit der Studien wies 2 oder 3 Verzerrungsrisiken auf. Drop-out-Raten von über 15\% während der Durchführung der Studien stellen eine Einschränkung in der Generalisierbarkeit der Ergebnisse dar und weisen potenziell auf eine geringere Akzeptanz bei den Teilnehmern hin. Kurze Follow-up-Zeiträume limitieren die Interpretierbarkeit der Ergebnisse. Langzeituntersuchungen sind jedoch besonders bedeutsam für die Bewertung der Effektivität hinsichtlich des präventiven Erfolgs. In 3 der 11 Programme wurde nicht untersucht, ob die Präventionsmaßnahme langfristig einen Einfluss auf die Entwicklung von Risikofaktoren für Essstörungen hat. Eine erwartete Limitation stellen die unterschiedlichen Stichprobencharakteristiken und -größen sowie verschiedene Messinstrumente der einzelnen Studien dar. Um dennoch die Vergleichbarkeit der Ergebnisse zu gewährleisten, wurden Effektstärken berechnet. Darüber hinaus dient die Einschätzung der Verzerrungsrisiken zur realistischeren Interpretation der Ergebnisse. Die Generalisierbarkeit der Evaluationsstudien ist zudem eingeschränkt, da nur 7 Studien ein randomisiert kontrolliertes Design aufwiesen. 
Ein Hauptkritikpunkt integrativer Forschungsarbeiten stellt die mögliche Publikationsverzerrung aufgrund unpublizierter Studien dar. In der vorliegenden Arbeit wird dieses Risiko als gering eingeschätzt. Es erfolgte eine umfangreiche Suche nach unpublizierter Literatur, welche im Einschluss von 5 nicht veröffentlichten Arbeiten in dieses Review resultierte.

\section{Implikationen für zukünftige Forschung}

(1) Die Ergebnisse verdeutlichen, dass Programme mit sekundärpräventivem Ansatz höhere Effekte erzielen. Dies sollte jedoch nicht in einer alleinigen Fokussierung auf diesen Präventionsbereich resultieren. Die geringen Effekte bei primärpräventiven Programmen sind aufgrund noch niedriger Ausprägungen der Risikofaktoren in der Stichprobe ableitbar. Bei sekundärpräventiven Programmen besteht hingegen das Risiko, dass die Intervention zu spät stattfindet und nicht alle Personen identifiziert werden, die von dem Programm profitieren könnten. Darüber hinaus stellen primärpräventive Programme trotz geringerer statistischer Wirksamkeit kosteneffektive und langfristig bedeutungsvolle Maßnahmen dar [70].

(2) Eine entscheidende Limitation der Studien ist häufig der kurze Untersuchungszeitraum. Dies ist besonders bei primärpräventiven Programmen relevant. Die Maßnahmen werden zeitlich häufig Jahre vor dem durchschnittlichen Erkrankungsalter durchgeführt. Kurzfristige statistische Effekte sind daher unwahrscheinlich [68]. Die Präventionseffekte sind somit auch erst mit deutlicher Verzögerung erfassbar. Daher sind Langzeituntersuchungen von zentraler Bedeutung. Die meisten Studien untersuchen jedoch nur die kurzfristige Wirksamkeit auf die Zielvariablen, was zu einer Unterschätzung der Präventionseffekte führen kann [71,72]. Ein Qualitätsmerkmal von Präventionsprogrammen ist daher die Evaluation nach mehreren Jahren, welches das Primärpräventionsprogramm PriMa aufweist [36]. Zukünftige Forschung sollte auf diesen Qualitätsstandard besonderen Wert legen. Dies würde Aussagen über die Wirksamkeit von Präventionsmaßnahmen hinsichtlich der Senkung von Inzidenzraten ermöglichen und somit eine starke Argumentationsgrundlage zur Planung von Präventionsstrategien bilden.

(3) Internationale Forschung weist auf sehr gute Evidenz primärer Präventionsprogramme hin, welche die Medienkompetenz fördern $[14,15]$. Nicht alle deutschsprachigen Präventionsprogramme widmen sich explizit der Förderung der Medienkompetenz. Die stärkere Einbeziehung dieser Komponente in zukünftige Strategien erscheint vor dem Hintergrund internationaler Literatur und der zunehmenden Digitalisierung der Gesellschaft sinnvoll.

(4) Vielversprechende Ergebnisse lieferten die Studien zu dem internetgestützten Programm Student Bodies. Auch internationale, internetbasierte Präventionsmaßnahmen für AN und BN erwiesen sich als wirksam und bieten eine kostengünstige Alternative zu schulbasierten Programmen. Besonders vorteilhaft erscheint die Anonymität, welche potenziell die Hemmschwelle senkt, externe Hilfe aufzusuchen [73]. Bei der Entwicklung oder Modifikation von sekundärpräventiven Programmen erscheint somit die Nutzung internetbasierter Strategien empfehlenswert.
(5) Als hilfreich erweist sich besonders die Stärkung der Identitätsentwicklung innerhalb der Präventionsprogramme, da dies in Zusammenhang mit einem höheren Selbstwert steht [74]. In diesem Zusammenhang kritisieren Corning und Heibel die häufig einseitige Fokussierung auf den Körperselbstwert innerhalb von Präventionsprogrammen. Sie schlagen eine Schwerpunktsetzung auf die Förderung einer umfassenden Identitätsentwicklung vor, was als entscheidender protektiver Faktor bei Essstörungen verstanden wird [75].

(6) In den Evaluationen der deutschsprachigen Programme fehlt die Untersuchung der Internalisierung des Schlankheitsideals. Dieser Risikofaktor hat sich bereits als relevante Einflussgröße erwiesen $[6,12,76]$ und sollte daher in künftigen Studien Beachtung finden.

\section{Implikationen für die Praxis}

Für die Auswahl eines geeigneten Präventionsprogramms sollten sich Lehrkräfte, klinisch Tätige und andere Interessierte an folgenden Punkten orientieren.

1) In welchem Setting (z. B. Schule, Internet) und für welche Zielgruppe (z. B. Kinder ab der 6. Klasse, junge Frauen ab 18 Jahren) soll das Programm eingesetzt werden? (

2) Welche Inhalte sollen transportiert werden und welche Risikooder Schutzfaktoren sollen beeinflusst werden? Inhaltlich gibt es bei vielen Programmen Überlappungen, so werden z. B. die Themen Schönheitsideale und gesunde Ernährung in nahezu allen Programmen aufgegriffen. Unterschiedlich ist jedoch die methodische Umsetzung. Alle Programme legen Wert auf eine interaktive Vermittlung der Inhalte über verschiedene Methoden, jedoch wird bspw. bei einigen Programmen mehr Fokus auf Gruppendiskussionen gelegt, bei anderen mehr Wert auf kognitiv-behaviorale Übungen. Es kann angenommen werden, dass Komponenten wie Medienkompetenz durch die zunehmende Digitalisierung des Alltags an Bedeutsamkeit gewonnen haben. Eine fortlaufende Wirksamkeitsprüfung und ggf. Adaption der Programme an das Zeitalter der modernen Jugendlichen ist daher ratsam.

3) Wie ist der zeitliche Umfang? Alle evaluierten Programme bestehen aus mehreren Sitzungen. Dies ist wichtig, um die Themen und Inhalte elaboriert und detailliert zu besprechen. Aus den Validierungsstudien ergab sich der Hinweis, dass längere Programme tendenziell wirksamer sind.

4) Welche Programme haben sich als wirksam erwiesen und sind ausreichend evaluiert? 11 der 22 identifizierten Programme sind wissenschaftlich evaluiert worden. Prinzipiell sollte der Einsatz nicht evaluierter Programme vermieden werden. Der positive Nutzen eingesetzter Programme sollte belegt sein und eventuell schädliche Programmwirkungen ausgeschlossen werden. Insgesamt wurde eine schädliche Programmwirkung bei keinem der evaluierten Programme berichtet. Dennoch muss hier die Publikationsverzerrung berücksichtigt werden, da möglicherweise negative Ergebnisse nicht publiziert wurden.

5) Wie sind die Programme verfügbar? Im letzten Schritt ist die Verfügbarkeit der Programme, der Materialien und eventueller Schulungen ein entscheidendes Auswahlkriterium. Nur so kann eine langfristige Verbreitung und Implementierung in den Präventionsalltag gewährleistet werden. 
Das vorliegende systematische Review verfolgte das Ziel, eine Übersicht bestehender deutschsprachiger Präventionsprogramme und deren Wirksamkeit bereitzustellen. Die Integration mehrerer Evaluationsstudien zu einem Präventionsprogramm sowie die Beachtung unpublizierter Literatur erhöht die Aussagekraft des systematischen Reviews. Insgesamt ist die Evidenz vielversprechend: Alle Studien zeigten sich wirksam in der Veränderung mind. einer Zielvariablen zur Postmessung in der Gesamtstichprobe oder in der Risikogruppe. Falls Follow-up Daten erhoben wurden, so blieb der Effekt bei mind. einem Outcome auch zu diesem Zeitpunkt erhalten. Gute Wirksamkeit zeigte sich für die Variablen Gewichts- und Figursorgen, (Körper-) Selbstwert und Schlankheitsstreben. Außerdem gut belegt wurde die positive Wirkung der Programme auf die Variable essstörungsspezifisches Wissen. Psychoedukative Anteile und erfolgreiche Wissensvermittlung allein erwiesen sich jedoch nicht als ausreichend wirksam in der Prävention von Essstörungen [11]. Inkonsistente Ergebnisse zeigten sich hinsichtlich der Wirksamkeit der Programme auf aufälliges Essverhalten und Figurunzufriedenheit. Um reliable Aussagen bezüglich der Wirksamkeit von Präventionsstrategien zu treffen, sind weitere langjährige Untersuchungen von zentraler Bedeutung.

\section{FAZIT FÜR DIE PRAXIS}

Es liegen 11 wissenschaftlich evaluierte, deutschsprachige Präventionsprogramme mit primär- und sekundärpräventiven Ausrichtungen vor, die wirksam in der Reduktion von Risikofaktoren sowie Förderung von Schutzfaktoren in verschiedenen Settings sind. Somit bietet die vorliegende Arbeit sowohl eine Übersicht für Anwender als auch einen Ausgangspunkt für weitere Forschungsansätze. Insbesondere Langzeituntersuchungen sollten dabei im Fokus stehen.

\section{Interessenkonflikt}

Die Autoren geben an, dass kein Interessenkonflikt besteht.

\section{Literatur}

[1] Treasure J, Claudino AM, Zucker N. Eating disorders. Lancet 2010; 375: 583-593

[2] Smink FRE, van Hoeken D, Oldehinkel A] et al. Prevalence and severity of DSM-5 eating disorders in a community cohort of adolescents. Int J Eat Disord 2014; 47: 610-619

[3] Hölling H, Schlack R. Essstörungen im Kindes- und Jugendalter. Erste Ergebnisse aus dem Kinder- und Jugendgesundheitssurvey (KiGGS). Bundesgesundheitsblatt, Gesundheitsforschung, Gesundheitsschutz 2007; 50: 794-799

[4] Nagl M, Jacobi C, Paul M et al. Prevalence, incidence, and natural course of anorexia and bulimia nervosa among adolescents and young adults. Eur Child Adolesc Psychiatry 2016; 25: 903-918

[5] Herpertz-Dahlmann B, Wille N, Holling $\mathrm{H}$ et al. Disordered eating behaviour and attitudes, associated psychopathology and healthrelated quality of life: results of the BELLA study. Eur Child Adolesc Psychiatry 2008; 17: (Suppl 1): 82-91
[6] Jacobi C, Hayward C, de Zwaan M et al. Coming to terms with risk factors for eating disorders: Application of risk terminology and suggestions for a general taxonomy. Psychol Bull 2004; 130: 19-65

[7] Keel PK, Forney KJ. Psychosocial risk factors for eating disorders. Int ] Eat Disord 2013; 46: 433-439

[8] Berger U. Primärprävention bei Essstörungen. Psychotherapeut 2006; 51: 187-196

[9] Smolak L, Thompson JK. Body image, eating disorders, and obesity in youth: Assessment, prevention, and treatment. 2nd ed.Washington, DC: American Psychological Association; 2009

[10] Ciao AC, Loth K, Neumark-Sztainer D. Preventing eating disorder pathology: common and unique features of successful eating disorders prevention programs. Curr Psychiatry Rep 2014; 16: 453

[11] Stice E, Shaw H. Eating disorder prevention programs: a meta-analytic review. Psychol Bull 2004; 130: 206-227

[12] Stice E, Shaw H, Marti CN. A meta-analytic review of eating disorder prevention programs: encouraging findings. Annu Rev Clin Psychol 2007; 3: 207-231

[13] Yager Z, Diedrichs PC, Ricciardelli LA et al. What works in secondary schools? A systematic review of classroom-based body image programs. Body Image 2013; 10: 271-281

[14] Le LK-D, Barendregt J], Hay P et al. Prevention of eating disorders: A systematic review and meta-analysis. Clin Psychol Rev 2017; 53: 46-58

[15] Watson H], Joyce T, French E et al. Prevention of eating disorders: A systematic review of randomized, controlled trials. Int J Eat Disord 2016; 49: 833-862

[16] Shaw $\mathrm{H}$, Stice E. The implementation of evidence-based eating disorder prevention programs. Eat Disord 2016; 24: 71-78

[17] Bauer S, Kindermann SS, Moessner M. Prävention von Essstörungen: Ein Überblick. Zeitschrift für Kinder-und Jugendpsychiatrie und Psychotherapie 2016; 44: 1-9

[18] Moher D, Liberati A, Tetzlaff J et al. Preferred reporting items for systematic reviews and meta-analyses: the PRISMA statement. Ann Intern Med 2009; 151: 264-269

[19] Pickhardt M, Richter F, Adametz L et al. German prevention programmes for eating disorders: A systematic review. In: http://www.crd.york.ac.uk/ PROSPERO/display_record.asp?ID = CRD42016042094 af ed, PROSPERO: International prospective register of systematic reviews; 2016

[20] Hedges LV, Olkin I. Statistical methods for meta-analysis. Orlando: Academic Press; 1985

[21] Cohen J. A Power Primer. Psychol Bull 1992; 112: 155-159

[22] Jones Bell M, Zeiler M, Kuso S et al. Healthy Teens @ School: Evaluating and disseminating transdiagnostic preventive interventions for eating disorders and obesity for adolescents in school settings. Internet Interventions in prep

[23] Buddeberg-Fischer B, Klaghofer R, Gnam G et al. Prevention of disturbed eating behaviour: A prospective intervention study in 14- to 19-year-old Swiss students. Acta Psychiatr Scand 1998; 98: 146-155

[24] Weigel A, Gumz A, Kastner D et al. Prävention und Versorgung von Essstorungen: Das Gesundheitsnetz Magersucht und Bulimie. Psychiatr Prax 2015; 42: S30-S34

[25] Weigel A, Gumz A, Uhlenbusch $N$ et al. Preventing eating disorders with an interactive gender-adapted intervention program in schools: study protocol of a randomized controlled trial. BMC Psychiatry 2015; 15: $1-9$

[26] Frankfurter Zentrum für Essstörungen. 2006; Body Talk. Im Internet: http://www.essstoerungen-frankfurt.de/bodytalk/anleitung.pdf; Stand: 07.07.2017

[27] Redeker M.BKK 2009;Bauchgefühl. Im Internet: http://www. bkk-bauchgefuehl.de/schule/fuer-schulen; Stand: 05.07.2017 
[28] Dietze-Cruse M, Fleischer H, Vogel N. 2011; Essenslust und Körperfrust - Leitfaden zur Prävention von Essstörungen in der Schule. In: Landesinstitut für Lehrerbildung und Schulentwicklung: SuchtPräventionsZentrum Hamburg. Im Internet: http://li.hamburg.de/contentblob/ 2833842/5273dae0fd1d3b60b0c1dcbed1576f00/data/pdf-essenslustund-koerperfrust-leitfaden-zur-praevention-von-essstoerungen-inder-schule.pdf; Stand: 05.07.2017

[29] Jacobi C, Beintner I. 2016; everyBody - Tailored online health promotion and eating disorder prevention for women at different risk stages. Im Internet: http://www.isrctn.com/ISRCTN13716228 ; Stand: 07.07.2017

[30] Schlevogt V. Das Präventionsprojekt JUGEND MIT BISS des Frankfurter Zentrums für Essstörungen (Abschlussbericht). In.Frankfurt am Main: Bundesministerium für Familie, Senioren, Frauen und Arbeit; 2002

[31] Bürger A.MaiStep- Mainzer Schultraining zur Essstörungsprävention [Dissertation]. Potsdam: Universität Potsdam; 2015

[32] Warschburger P, Helfert S, Krentz EM. POPS: A school-based prevention programme for eating disorders. J Public Health 2011; 19: 367-376

[33] Sowa M. Längerfristige Effekte der Programmwirkung des Schulprojektes PriMa zur Primärprävention von Magersucht [Unpublizierte Diplomarbeit]. Jena: Friedrich-Schiller-Universität Jena; 2006

[34] Berger U, Joseph A, Sowa M et al. Die Barbie-Matrix: Wirksamkeit des Programms PriMa zur Primärprävention von Magersucht bei Mädchen ab der 6. Klasse. Psychother Psychosom Med Psychol 2007; 57: 248-255

[35] Wick K, Brix C, Bormann B et al. Real-world effectiveness of a German school-based intervention for primary prevention of anorexia nervosa in preadolescent girls. Prev Med 2011; 52: 152-158

[36] Adametz L, Richter F, Strauss B et al. Long-term effectiveness of a school-based primary prevention program for anorexia nervosa: A 7-to 8-year follow-up. Eat Behav 2017; 25: 42-50

[37] Beintner I, Jacobi C, Taylor CB. Effects of an Internet-based prevention programme for eating disorders in the USA and Germany - A meta-analytic review. Eur Eat Disord Rev 2012; 20: 1-8

[38] Fritsche A, Schlenkrich C. Evaluation des Internetgestützten Präventionsprogramms Student Bodies zur Reduktion von Risikofaktoren für gestörtes Essverhalten an Mädchen der 8. bis 10. Klasse [Unpublizierte Diplomarbeit]. Dresden: Technische Universität Dresden; 2005

[39] Endruschat C, Gere M. Evaluation des Internetgestützten Präventionsprogramms Student Bodies zur Reduktion von Risikofaktoren für gestörtes Essverhalten an einer Risikostichprobe junger Studentinnen der TU Dresden [Unpublizierte Diplomarbeit]. Dresden: Technische Universität Dresden; 2006

[40] Jacobi C, Morris L, Beckers C et al. Maintenance of internet-based prevention: a randomized controlled trail. Int J Eat Disord 2007; 40: 114-119

[41] Gerhard E. Prävention von Essstörungen - Wirkungsevaluation des Schulprojektes "Torera" [Unpublizierte Diplomarbeit]. Jena: FriedrichSchiller-Universität Jena; 2006

[42] Berger U, Schaefer JM, Wick $K$ et al. Effectiveness of reducing the risk of eating-related problems using the German school-based intervention program, 'Torera', for preadolescent boys and girls. Prev Sci 2014; 15: $557-569$

[43] Dannigkeit N, Köster G, Tuschen-Caffier B. Ist primäre Prävention von Essstörungen langfristig wirksam? Ergebnisse zur Evaluation eines Trainingsprogramms an Schulen. Z Gesundheitspsychol 2005; 13: 79-91

[44] Lindenberg K, Kordy H. Wirksamkeit eines gestuften, internetvermittelten Ansatzes zur Prävention von Essstörungen bei Schülern der 7. bis 10. Klasse. Kindh Entwickl 2015; 24: 55-63
[45] Hötzel K, von Brachel R, Schmidt U et al. An Internet-based program to enhance motivation to change in females with symptoms of an eating disorder: a randomized controlled trial. Psychol Med 2014; 44: 1947-1963

[46] Bauer S, Moessner M, Wolf M et al. ES[S]PRIT - an Internet-based programme for the prevention and early intervention of eating disorders in college students. $\mathrm{Br}$ J Guid Counc 2009; 37: 327-336

[47] Köster G, Dannigkeit N, Tuschen-Caffier B. Indizierte Prävention von Essstörungen: Eine Pilotstudie zu einem ambulanten Gruppenprogramm. Z Gesundheitspsychol 2005; 13: 102-107

[48] Köster G. Indizierte Prävention von Essstörungen: Entwicklung und Evaluation eines Gruppenprogramms [Unpublizierte Dissertation]. Freiburg: Albert-Ludwigs-Universität; 2011

[49] Minarik C, Moessner M, Ozer F et al. Implementierung und Dissemination eines internet-basierten Programms zur Prävention und frühen Intervention bei Essstörungen. Psychiatr Prax 2013; 40: 332-338

[50] Moessner M, Özer F, Minarik C et al. ProYouth-ein Online-Programm zur Prävention und frühen Intervention bei Essstörungen. PiD 2013; 14: 74-76

[51] Bauer S, Papezova H, Chereches R et al. Advances in the prevention and early intervention of eating disorders: The potential of Internetdelivered approaches. Mental Health \& Prevention 2013; 1: 26-32

[52] Jacobi C, Voelker U, Trockel MT et al. Effects of an Internet-based intervention for subthreshold eating disorders: A randomized controlled trial. Behav Res Ther 2012; 50: 93-99

[53] Ohlmer R, Jacobi C, Taylor CB. Preventing symptom progression in women at risk for AN: Results of a pilot study. Eur Eat Disord Rev 2013; 21: $323-329$

[54] Moessner M, Minarik C, Ozer F et al. Effectiveness and Cost-effectiveness of School-based Dissemination Strategies of an Internet-based Program for the Prevention and Early Intervention in Eating Disorders: A Randomized Trial. Prev Sci 2016; 17: 306-313

[55] Tuschen-Caffier B. Essstörungen: Klassifikation und Diagnostik. In: Baumann UP M., (Hrsg.). Klinische Psychologie und Psychotherapie. Bern: Huber; 2005: 746-760

[56] Morgan JF, Reid F, Lacey JH. The SCOFF questionnaire: assessment of a new screening tool for eating disorders. Br Med J 1999; 319: 1467-1468

[57] Hilbert A, Tuschen-Caffier B, Ohms M. Eating disorders examination: A German version of the structured eating disorder interviews. Diagnostica 2004; 50: 98-106

[58] Thiel A, Jacobi C, Horstmann S et al. A German translation of the eating disorder inventory EDI-2. Psychother Psych Med 1997; 47: 365-376

[59] Childress AC, Brewerton TD, Hodges EL et al. The Kids' Eating Disorders Survey (KEDS): A study of middle school students. J Am Acad Child Adolesc Psychiatry 1993; 32: 843-850

[60] Killen JD, Taylor CB, Hayward C et al. Weight concerns influence the development of eating disorders: A 4-year prospective study. J Consult Clin Psychol 1996; 64: 936-940

[61] Kuhner C, Burger C, Keller F et al. Reliability and validity of the Revised Beck Depression Inventory (BDI-II). Results from German samples. Nervenarzt 2007; 78: 651-656

[62] Strauß B, Richter-Appelt H. Fragebogen zur Beurteilung des eigenen Körpers (FBeK). Göttingen: Hogrefe Verlag für Psychologie; 1996

[63] von Collani G, Herzberg PY. Eine revidierte Fassung der deutschsprachigen Skala zum Selbstwertgefühl von Rosenberg. Zeitschrift für Differentielle und Diagnostische Psychologie 2003; 24: 3-7

[64] Marsh HW. Self Description Questionnaire-III: SDQ III Manual. Macarthur: University of Western Sydney; 1990

[65] Hohler RR. Selbstwertschwankungen bei Bulimia Nervosa. Marburg: Tectum; 1997 
[66] Winzelberg A], Taylor CB, Sharpe T et al. Evaluation of a computermediated eating disorder intervention program. Int J Eat Disord 1998; 24: 339-349

[67] Dannigkeit N, Köster G, Tuschen-Caffier B. Prävention von Essstörungen-Ein Trainings-programm für Schulen. In: Röhrle B, (Hrsg.). Prävention und Gesundheitsförderung Bd 2. Tübingen: Deutsche Gesellschaft für Verhaltenstherapie; 2002: 151-171

[68] Wilksch SM. Where did universal eating disorder prevention go? Eat Disord 2014; 22: 184-192

[69] Passon AM, Gerber A, Schröer-Günther M. Wirksamkeit von schulbasierten Gruppeninterventionen zur Depressionsprävention. Kindh Entwickl 2011; 20: 236-246

[70] Horowitz JL, Garber J. The prevention of depressive symptoms in children and adolescents: A meta-analytic review. J Consult Clin Psychol 2006; 74: 401-415

[71] Wilksch SM. The last word: Where did universal eating disorder prevention go? Eat Disord 2014; 22: 184-192
[72] Stice E, Becker CB, Yokum S. Eating disorder prevention: Current evidence-base and future directions. Int J Eat Disord 2013; 46: 478-485

[73] Schlegl S, Burger C, Schmidt L et al. The potential of technology-based psychological interventions for anorexia and bulimia nervosa: a systematic review and recommendations for future research. J Med Internet Res 2015; 17: e85

[74] Kroger J. Marcia JE. The identity statuses: Origins, meanings, and interpretations. In: Schwartz SJ, Luyckx K, Vignoles VL, (Hrsg.). Handbook of identity theory and research. New York: Springer Science + Business Media; 2011

[75] Corning AF, Heibel HD. Re-thinking eating disorder prevention: The case for prioritizing the promotion of healthy identity development. Eat Disord 2016; 24: 106-113

[76] Jacobi C, Fittig E, Bryson SW et al. Who is really at risk? Identifying risk factors for subthreshold and full syndrome eating disorders in a high-risk sample. Psychol Med 2011; 41: 1939-1949 